\title{
Diagonal Direct Sub Pixel based Down Sampling Filter for Antialiasing the Image
}

\author{
Prachi Rohit Rajarapollu \\ MIT Academy of Engineering \\ Alandi, Pune, India
}

\author{
Vijay R. Mankar \\ Dy. Secretary, M.S. Board of \\ Tech. Education \\ Pune Regional Office. India
}

\begin{abstract}
A solitary sensor advanced camera needs demosaicing to remake a full shading picture. To demonstrate the high determination picture on the lower determination show, it should then be down examined. Demosaicing and downsampling are the two stages that impact one another. To begin with is, the shading bordering curios present in demosaicing might be seem bigger in consequent down-inspecting process. Then again, the subtle element evacuated amid the downexamining can't be recuperated in the demosaicing. Thus, it is vital to consider the demosaicing and down-examining handle at the same time. In this paper, utilization of recurrence space investigation to clarify what happens in sub pixel-based down inspecting and why it is conceivable to accomplish a higher obvious determination is done. By recurrence space investigation and perception, the cut off recurrence of the lowpass channel for sub pixel-based obliteration can be successfully developed past the Nyquist recurrence utilizing a novel against associating channel. Applying the proposed channels to two existing sub pixel down inspecting plans called direct sub pixel-based down sampling (DSD) and corner to corner DSD (DDSD), we get two enhanced plans, i.e., DSD in based on frequency -domain analysis(DSD-FA) and DDSD in view of recurrence area examination (DDSDFA). Trial results check that the proposed DSD-FA and DDSD-FA can give predominant results, contrasted and existing sub pixel or pixel-based down examining techniques.
\end{abstract}

\section{General Terms}

Antialiasing, Image down sampling, frequency sampling, direct sub pixel-based down sampling (DSD)

\section{Keywords}

Down sampling, frequency analysis, sub pixel rendering.

\section{INTRODUCTION}

A sign pixel on a shading liquid-crystal display (LCD) comprises of a few essential colors, which are ordinarily three colored stripes requested (contingent upon the presentation) either as blue, green, and red (BGR), or as red, green, and blue (RGB). The shaded stripes are called sub pixels. The shades of the three sub pixels are combined to show up as a solitary shading to the human visual System(HVS) because of the obscuring by the optics and the spatial mix by the nerve cells in the human eyes [1]-[3]. In spite of the fact that the sub-pixels are effortlessly obvious when seen at a nearby separation (especially with the assistance of an amplifying glass), they are not separately noticeable past a specific separation. In any case, analysts found that, by controlling the subpixel benefits of neighboring pixels, it is conceivable to microshift the clear position of a line and/or achieve higher edge sharpness. Methods that take this into account are called sub pixel rendering algorithms [4].

\section{WHAT IS ANTI-ALIASING?}

The solution to the problem of aliasing and missing pixels is named 'anti-aliasing'. This is a term which can apply to both audio and visual signals to describe the representation of a high-resolution signal at a lower resolution. In the case of displayed images, this technique involves applying a complex formula to ascertain and execute the in-filling between the lighter and darker pixels of an image with grey or mixed colours and converting alias pixels to grey, this effectively blurs or smoothes the edge between light and dark areas of an image. When viewing this type of conversion from a distance, the eye sees a more consistent and clearer joined-up image as opposed to the previously pixilated or broken up version. However it is an extremely productive way to help improve the readability of an image as the brain essentially makes up for the missing pixel data by subconsciously 'filling in the blanks' as a user reads images presented in this way [4]. The fundamental problem to be tackled when developing antialiasing techniques is to optimize color changes whilst keeping the time to do this process to a minimum. Also, and not to do it so much as to add too-much grey so that the images appear blurred which can result in worse effects, perhaps even eye strain. The time issue is well illustrated by considering that a typical image may have in excess of three million pixels and to perform even simple anti-aliasing may take several times number of calculations [5]. The duration of an anti-aliasing process may therefore take several seconds, or perhaps even minutes difficult to accept in our speed driven world.

\section{DIFFERENT ANTI-ALIASING TECHNIQUES}

Antialiasing can be done by using following some of the methods,

- Adaptive post-filtering

- Texture antialiasing

- $\mathrm{G}-$ Buffer based antialiasing

- $\quad$ Randomized antialiasing

- Poly-phase antialiasing

- Heat Kernel Smoothing algorithm

- $\quad$ Edge and Corner Enhancing Smoothing

In this paper, image down sampling utilizing sub pixel systems to accomplish more honed images for little LCDs. Such an issue exists when a high-determination images or video is to be shown on low determination show terminals. For instance, while advanced images are typically caught at high resolutions (e.g., 10 megapixels), a large number of them would be shown on LCD PC screens, photograph edges, or little LCD screens on cell telephones or individual 
computerized aides, which have impressively bring down resolutions (e.g., 0.8 megapixels on SVGA or 0.2 megapixels on some PDAs). A comparable circumstance exists for recordings, where full-superior quality (HD, 1920 1080) TV or full-HD films in Blue-beam might be seen on HD-prepared (1366 768) or standard-definition (720 576 or 720 480) TVs or screens. To view high-determination images/recordings on low-determination shows, a down inspecting system is required. A basic way called Direct Pixel-based Down examining (DPD) in this paper is to perform down selecting so as to test one out of each pixels. It can acquire serious associating antiques in districts with high spatial recurrence [such as staircase ancient rarities and broken lines, as appeared. An enhanced technique is called Pixel-based Down sampling with an Anti-associating Filter (PDAF), in which the counter associating channel is connected before DPD. It stifles associating antiquities to the detriment of obscuring the images, as just low-recurrence data can be held in the process [10], [11]. Note that neither the DPD nor the PDAF bring about shading artifacts.

\section{AIM \& OBJECTIVES}

Aliasing in computer-synthesized images not only limits the realism of the images, but also affects the user's concentration. The goal of color image smoothing and de-noising is to remove spurious details and/or noise for a given possibly corrupted image, while maintaining essential features such as edges. Effective and fast processing of digital images has not always been easy when the collection of images grow into thousands. e.g. finding an image of a bird from an image taken from a long distance without fading the image is a difficult task

\section{RELATED WORK}

\subsection{Direct Sub Pixel-Based Down Sampling Based On Frequency Analysis ( DSD- FA).}

For effortlessness, we accept that an input high-resolution image $\mathrm{L}$ (which means extensive) of size $\mathrm{M}^{*} \mathrm{~N}$ is to be down sampled to a low-resolution image $\mathrm{S}$ (which means little ) of size $M^{*} N$, to be shown on $M^{*} N$ a device, where $M=3 m$ and $\mathrm{N}=3 \mathrm{n}$. (Note that if is not of size $3 \mathrm{~m} * 3 \mathrm{n}$, i.e., the down sample proportion is not 3, we can utilize general insertion or devastation strategies to resize to be $3 \mathrm{~m} * 3 \mathrm{n}$.) In this paper, we will use(i,j) to record $\mathrm{L}$ and (i',j')to file $\mathrm{S}$ such that(Rij,Gij,Bij) is the (i.j)th pixel of $L$ and(Tij,gij,bij) is the (i',j')th pixel of $S$. Daly and Kovvuri [16] proposed a straightforward sub pixelbased down sampling plan that we call DSD in this paper. DSD copies the red, green, and blue segments then again in the level course. DSD duplicates the red, green, and blue segments (i.e., the three sub pixels) of the pixel from three distinct pixels in $\mathrm{L}$, such that ,rij=R3i-1,3j'- 2, gi',j'=G3i'- 1,3j'1 , and bi'j'=B3i'- $1,3 j^{\prime}$, , while the associating antiques in DPD cause the grass to be "broken", the DSD fills in the continuous in the grass, making it ceaseless and sharp to the detriment of presenting undesirable shading bordering ancient artifacts.

\subsection{Diagonal Direct Sub Pixel Based Down Sampling (DDSD)}

In the sub pixel font style rendering innovation is talked about. All the current techniques for subpixel based downsampling perform horizontal subsampling. This is on the grounds that the red, green, blue subpixels of a run of LCD display are in a horizontal level way. There are regularly no smooth areas or area with smooth surface because of the horizontal subsampling in DSD. The subpixel-based down- sampling should be possible in horizontal, diagonal, or antidiagonal direction. (It doesn't bode well to test in vertical bearing as RGB subpixels are organized in level way.)The test is finished by down-sampling a fake huge image with consistent line width utilizing direct pixel-based downsampling (DPD), direct sub pixel-based down-sampling (DSD), and diagonal direct sub pixel-based downsampling(DDSD) method. DSD and DDSD are fundamentally the same aside from that DSD subsamples in the horizontal direction while DDSD subsamples in the diagonal direction. The straight-forward DPD gives a image with unpredictable line spacing, which is bad. Both DSD and DDSD save the line normality for lines in 3 directions at the cost of color fringing artifacts . They have no impact on the fourth direction (even for DSD and slanting for DDSD). In [9], DPD and PDAF don't bring about the color artifacts. In the existing paper, the horizontal lines happen more frequently than diagonal lines when all is said in done and along these lines presume that DDSD might be more helpful than DSD plainly clarifies the DDSD. At that point the frequency domain analysis apparatus is utilized to demonstrate that the cut-off frequency of the low-pass filter for JDSD can be successfully expanded past the Nyquist frequency , bringing about much more honed down-tested pictures. In [10], hostile to associating channel is connected in the down sampled image.

\subsection{Algorithm for super sampling}

- To generate the original image, we need to consider a region in the virtual image. The extent of that region determines the regions involved in the low pass operation. This process is called convolution.

- After we obtain the virtual image which is at a higher resolution, the pixels of the final image are located over super pixels in the virtual image. To calculate the value of the final image at $(\mathrm{Si}, \mathrm{Sj})$, we place the filter over the super image and compute the sum of the filter weights and the surrounding pixels. An adjacent pixel of the final image is calculated by moving the filter S superpixels to the right. Thus the step size is same as the scale factor between the real and the virtual image.

- Filters combine samples to compute a pixel's color. The weighted filter shown on the slide combines nine samples taken from inside a pixel's boundary. Each sample is multiplied by its corresponding weight and the products are summed to produce a weighted average, which is used as the pixel color. In this filter, the center sample has the most influence. The other type of filter is an un weighted filter. In an un-weighted filter, each sample has equal influence in determining the pixel's color. In other words, an un weighted filter computes an un weighted average.

- The spatial extent of the filter determines the cutoff frequency. The wider the filter, the lower is the cutoff frequency and the more blurred is the image.

- The options available in super sampling are

- The value of $\mathrm{S}$ - scaling factor between the virtual and the real images.

- The choice of the extents and the weights of the filter 
As far the first factor is concerned, higher the value, the better the result is going to be. The compromise to be made is the high storage cost.

\subsection{Existing System}

Demosaicing and down-sampling are incorporated together for single sensor bayer images utilizing bicubic strategy, because of which the computational complexity nature is fundamentally decreased. The bicubic technique is straightforwardly connected in Bayer area, without the procedure of demosaicing. This strategy utilizes all its encompassing neighbor pixels to compute the interpolated value to keep up the subtle element of the image. Programming results show that, the proposed strategy accomplishes unrivaled execution change regarding computational multifaceted nature. Concerning visual quality, this proposed technique is more viable in safeguarding high recurrence points of interest which prompts much more keen and clearer results.

\section{METHODOLOGY}

Conventional computer, software and hardware systems include graphics systems or subsystems that interact with data and commands to generate an image consisting of a plurality of pixels. One of the ways to define an image is by its underlying mathematical representation, such as a function that defines a plurality of curves or polygons, 3D models, textures, or any combination thereof. In this case, the image is produced by "sampling" the underlying representation. In this proposal the research work proposed is based on super sampling algorithm. This is done by obtaining a color of each pixel by evaluating the underlying representation at least once at coordinates corresponding to each pixel. "Sampling" is a conversion of a continuous-space signal (an image function) into a discrete-space signal (a plurality of pixels). The above underlying mathematical representation of the image is called an image function. Since the process of generating an image which is defined by an image function involves sampling, unwanted effects such as "aliasing" may appear in the image. Aliasing appears as jaggedness, unevenness or Moire patterns that are especially visible in the areas of the image corresponding to discontinuities in the original continuousspace signal, i.e., the image function. Further, aliasing is caused by frequencies which exceed the Nyquist limit. The Nyquist limit specifies that the original signal can be appropriately reconstructed from samples only if the sampling frequency is at least twice the maximum frequency of the original signal. Since discontinuities in the original signal create infinitely high frequencies, aliasing is most apparent at the boundaries between continuous regions of the image function. Examples of such boundaries are edges between polygons in a 3D model, conditional statements inside a shader code, or contours of a 2D polygon or curve. Hence, for the boundaries to appear smooth, anti-aliasing needs to be applied. This can include evaluating the image function multiple times per pixel. Conventional anti-aliasing techniques apply anti-aliasing to every pixel of the image. In other words, the anti-aliasing applies to continuous regions and discontinuities equally. This is inefficient, because continuous regions do not benefit from anti-aliasing. The result is that continuous areas will appear the same to a human eye as they were before the anti-aliasing was applied. On the other hand, the boundaries of the continuous regions of the image will benefit from the anti-aliasing. Hence, there is a need for a system and a method capable of alleviating expensive anti-aliasing techniques that anti-alias both continuous regions and discontinuities of the image function.
Further, there is a need for a system and a method that selectively applies anti-aliasing to discontinuities of the image function.

The method includes the steps of a) rendering a non-antialiased image having a region map, wherein the region map further includes at least one continuous region; $b$ ) determining at least one boundary of the at least one continuous region; and c) anti-aliasing the at least one boundary to generate an anti-aliased image. In an alternate embodiment, the present invention is a method for generating an image of a model. The method includes the following steps: a) projecting a model into image space; $b$ ) identifying at least one continuous region based on said projecting; c) determining at least one boundary of the at least one continuous region; and d) generating an anti-aliased image of the model, wherein generating further includes anti-aliasing the at least one boundary. In yet an alternate embodiment, the proposed invention is a method for anti-aliasing an image. The method includes the following steps: a) generating a non-anti-aliased image defined using an image function; b) locating at least one continuous region defined using the image function, wherein the at least one continuous region has at least one boundary; and c) generating an anti-aliased image, wherein generating further includes anti-aliasing the at least one boundary.

\section{PROPOSED SYSTEM}

The present method relates to computer graphics. Specifically, the present invention relates to image synthesis, image generation and visualization. The proposed method allows faster generating of images having anti-aliased (or smooth) edges. In an embodiment, the proposed method uses an image function in the form of color $=f(x, y)$ to generate anti-aliased images, where $\mathrm{x}$ and $\mathrm{y}$ are defined in an image coordinate space. This generation is accomplished by generating a nonanti-aliased image, determining continuous regions within the image function, projecting the regions into an image space, applying an edge-finding convolution to the projection to find pixels overlapping with the edges between continuous regions, and applying anti-aliasing to those pixels only. For this purpose the image function is modified to be capable of identifying its continuous regions. One of the advantages of the method is that anti-aliasing is applied only to the above pixels instead of being applied to every pixel in a final image which is going to increase the processing speed. Therefore, a significant amount of time is saved. To make the image smoother and allow it to have undistorted edges, anti-aliasing is applied. The anti-aliasing is applied to the edges, Solid areas do not benefit from application of anti-aliasing, because they do not contain discontinuities caused by conditional statements since these areas are evaluated by following the same path through conditional statements in the image function. Further, because in many cases solid areas occupy most of the image, and the modifications to the image function which are proposed in this report are relatively computationally inexpensive, limiting the application of antialiasing process to the boundaries of continuous regions can save a lot of expense and time. As such, the final anti- aliased image is rendered faster. The system flowchart has been shown in fig 01 . 


\section{SYSTEM ARCHITECTURE}

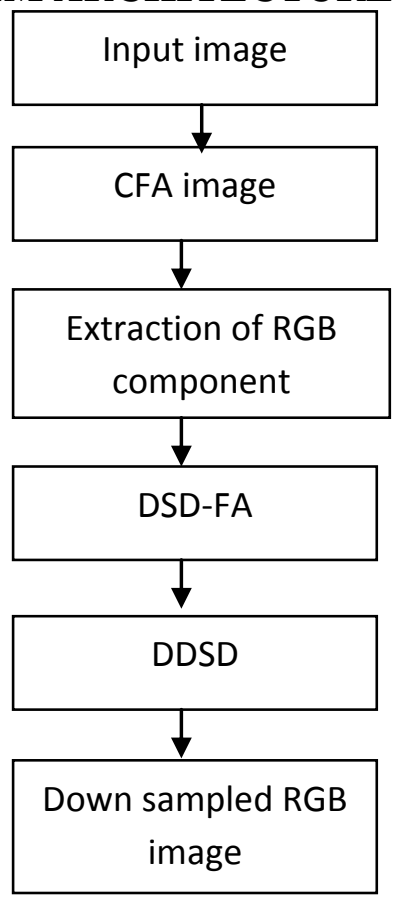

Fig. 01 System Flowchart

\section{RESULT ANALYSIS}

After implementation of different filters like, DDSD, DPD and DSD, the observations has been find out with different combinations like, DDSD butter-worth, Gaussian, and ideal. After that the result has been analysed with DPD filter in three different combination like, DPD Butter-worth, Gaussion and ideal. Finaly implementation of DSD filter has been done and result has been observed wth Butterworth, Gaussion and ideal filter. The comparion of all the filters has been given in table 01 .

\section{Comparison between filters}

\begin{tabular}{|c|c|c|c|}
\hline Parameters & Butterworth & Gaussian & Ideal \\
\hline S/N values & 93 & 80 & 76 \\
\hline Weight & 95 & 86 & 73 \\
\hline
\end{tabular}

Graphical representation of result has been shown in the following fig. 01

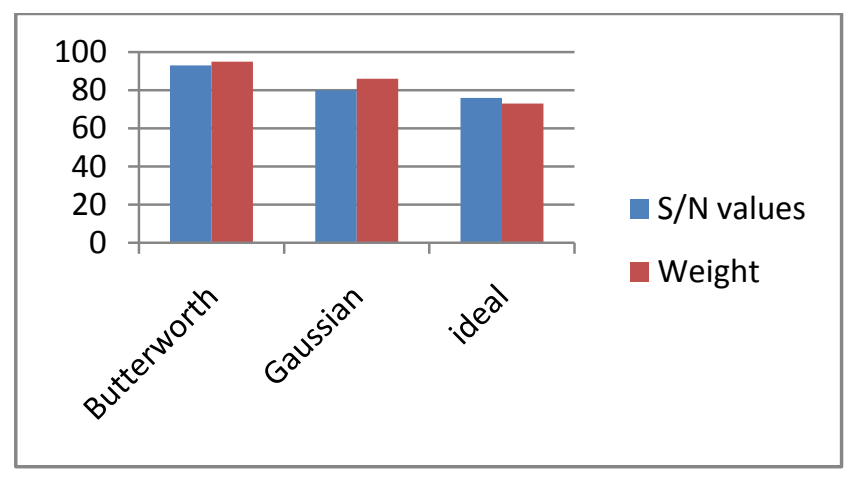

Fig. 01 Graphical representation of result

Results has been shown in fig. 02,03 and 04

\section{CONCLUSION}

In this paper, utilization of frequency domain analysis has been done to clarify the aliasing behavior of a several down sampling plans, i.e., DPD (pixel-based), DSD, and DDSD (sub pixel-based), and why it is vital to accomplish a higher clear determination. By frequency observation and analysis, the cut off frequency of the antialiasing filter for DSD and DDSD can be successfully augmented past the Nyquist frequency using novel antialiasing filter. Applying the proposed filter to DSD and DDSD, analysis gives two enhanced plans: DSD-FA and DDSD-FA. Test results confirm that the proposed DSD-FA and DDSD-FA can give better results thought about than existing sub pixel or pixel-based down examining techniques.

\section{ACKNOWLEDGMENT}

We might want to thank the analysts and also distributers for making their assets accessible. We additionally appreciative to commentator for their significant recommendations furthermore thank the school powers for giving the obliged base and backing.

\section{REFERENCES}

[1] Y. Amano, "A flat-panel TV display system in monochrome and color," IEEE Trans. Electron. Devices, vol. ED-22, no. 1, pp. 1-7, Jan. 1975.

[2] T. Benzschawel and W. E. Howard, "Method of and apparatus for displaying a multicolor image," U.S. Patent 5341 153, Aug. 23, 1994.

[3] L. M. Chen and S. Hasegawa, "Influence of pixelstructure noise on image resolution and color for matrix display devices," J. Soc. Inf. Display, vol. 1, no. 1, pp. 103-110, Jan. 1993.

[4] P. Barten, "Effects of quantization and pixel structure on the image quality of color matrix displays," in Proc. IEEE Int. Conf. Display Res., 1991, pp. 167-170.

[5] ClearType information [Online]. Available: http://www.microsoft. com/typography/cleartypeinfo.mspx.

[6] S. Gibson, Sub-pixel font rendering technology [Online] Available: http://www.grc.com/cleartype.htm.

[7] M. A. Klompenhouwer, G. De Haan, and R. A. Beuker, "Subpixel image scaling for color matrix displays," $J$. Soc. Inf. Display, vol. 11, no. 1, pp. 99-108, Mar. 2003.

[8] J.-S. Kim and C.-S. Kim, “A filter design algorithm for subpixel rendering on matrix displays," in Proc. 15th EUSIPCO, 2007, pp. 1487-1491.

[9] S. Daly, "Analysis of subtriad addressing algorithms by visual system models," in SID Int. Symp. Digest Tech. Papers, 2001, vol. 32, pp. 1200-1203.

[10] R. C. Gonzalez and E. W. Richard, Digital Image Processing. Englewood Cliffs, NJ: Prentice-Hall, 2005.

[11] P. S. R. Diniz, Digital Signal Processing. Cambridge, U.K.: Cambridge Univ. Press, 2010.

[12] J. C. Platt, "Optimal filtering for patterned displays," IEEE Signal Process. Lett., vol. 7, no. 7, pp. 179-181, Jul. 2000.

[13] C. Betrisey, J. F. Blinn, B. Dresevic, B. Hill, G. Hitchcock, B. Keely, D. P. Mitchell, J. C. Platt, and T. 
Whitted, "Displaced filtering for patterned displays," in Proc. SID Int. Symp. Digest Tech. Papers, 2000, vol. 31, pp. 296-301.

[14] D. S. Messing and S. Daly, "Improved display resolution of subsampled colour images using subpixel addressing," in Proc. IEEE ICIP, 2002, vol. 1, pp. I-625-I-628.
[15] D. S. Messing, L. Kerofsky, and S. Daly, "Subpixel rendering on nonstriped colour matrix displays," in Proc. IEEE ICIP, 2003, vol. 2, pp. II-949-II-952.

[16] S. J. Daly and R. R. K. Kovvuri, "Methods and systems for improving display resolution in images using subpixel sampling and visual error filtering," U.S. Patent 09/735 424, Aug. 19, 2000.

\section{APPENDIX}

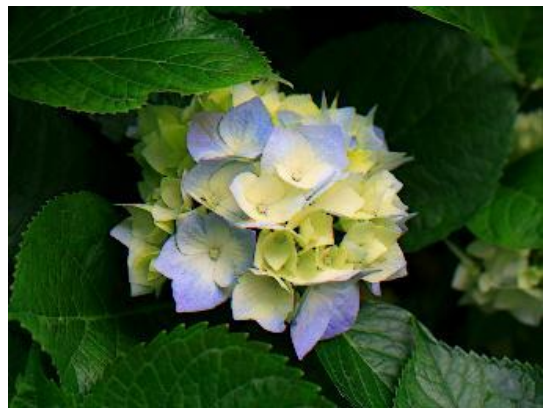

(a)

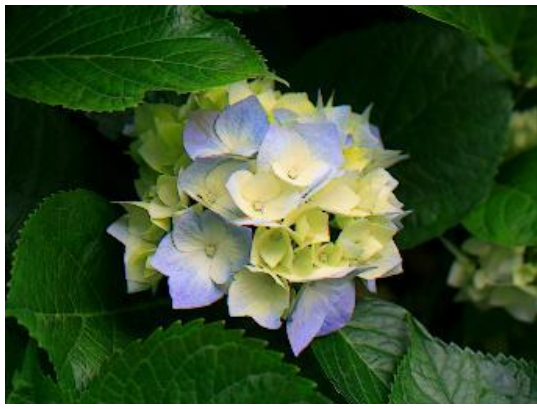

(b)

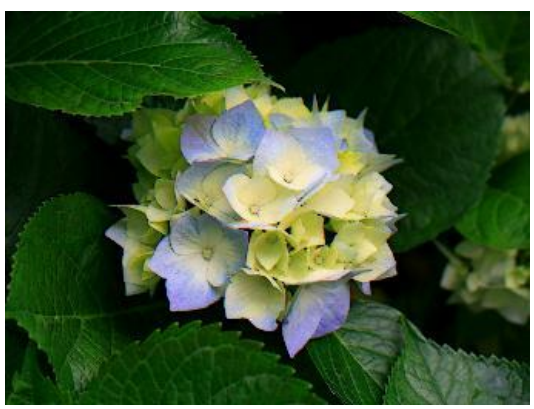

(c)

Figure 02 Direct diagonal based sub-pixel down sampling (a) DDSD butter-worth filter (b) DDSD Gaussian filter (c) DDSD ideal

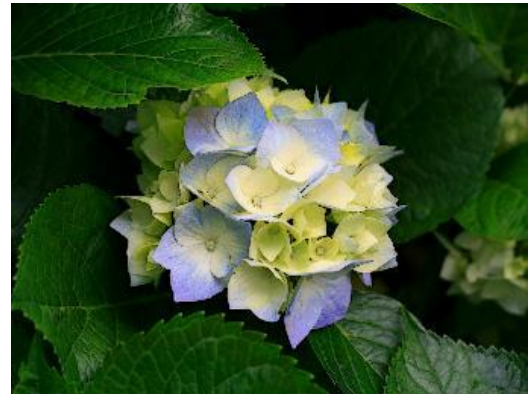

(a)

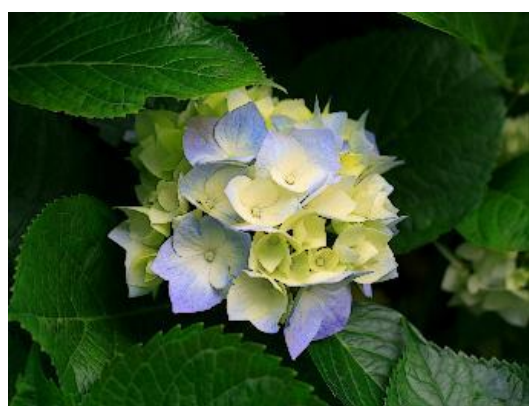

(b)

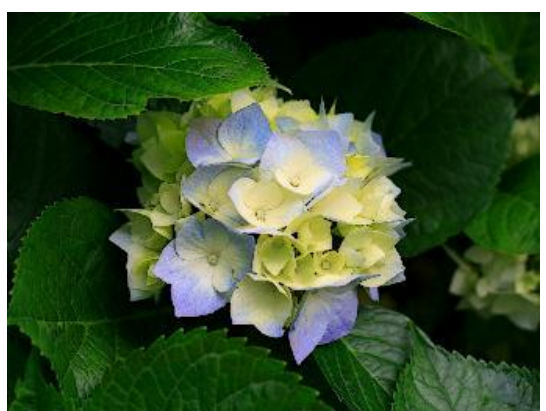

(c)

Figure 03 Direct pixel based down sampling (a) DPD butter-worth filter (b) DPD Gaussian filter (c) DPD ideal filter

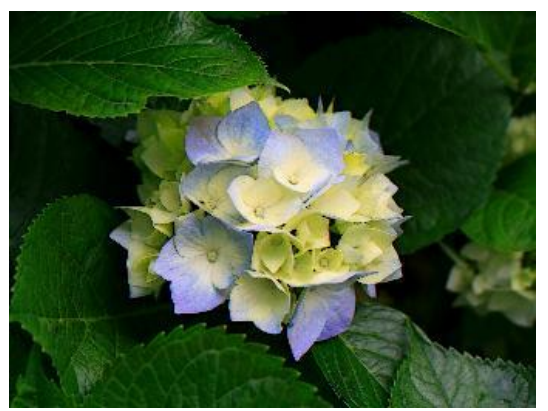

(a)

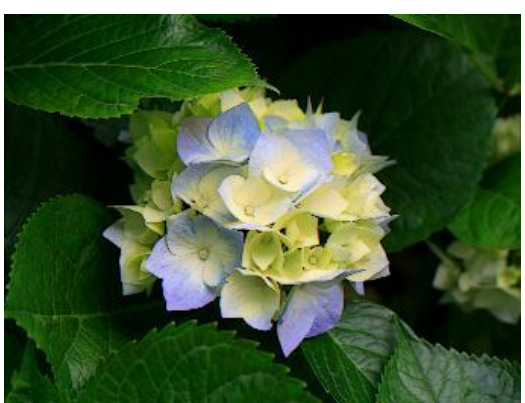

(b)

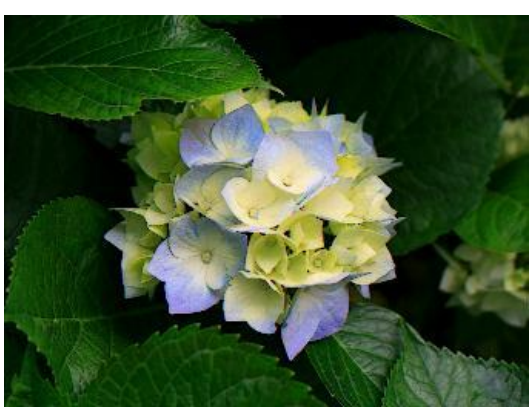

(c)

Figure 04 Direct sub-pixel based down sampling (a) DSD butter-worth filter (b) DSD Gaussian filter (c) DSD ideal filter 